\title{
An Enhanced Control Technique for the Elimination of Residual Vibrations in Flexible-Joint Manipulators
}

\author{
Caglar Conker ${ }^{1, *}$ - Ali Kilic ${ }^{2}$ - Selcuk Mistikoglu1 - Sadettin Kapucu² - Hakan Yavuz ${ }^{3}$ \\ ${ }^{1}$ Mustafa Kemal University, Faculty of Engineering, Turkey \\ 2 Gaziantep University, Faculty of Engineering, Turkey \\ ${ }^{3}$ Cukurova University, Faculty of Engineering, Turkey
}

One method used to reduce or eliminate residual vibrations is to modify the input signal by using previously determined system parameters. In order to eliminate the residual vibration completely, these system parameters must be very accurately determined. In real systems, achieving such accuracy may not always be possible. To address this problem and to provide a solution, a new residual vibration elimination method is introduced in this study, which has proven to be useful especially in cases of uncertain parameters of estimated or predicted systems. It is shown that the technique is capable of handling high levels of uncertainty and is able to successfully eliminate or reduce residual vibrations in flexible systems. In this approach, the desired position of the system is primarily divided into two equal parts, and the generated input signal is used to eliminate vibration. This study presents theoretical and experimental results of the techniques applied to a flexible mechanical system; a comparative study of robustness performance is also provided. Simulation and experimental results show that the oscillations are considerably decreased with a high degree of robustness in the presence of uncertainty regarding system parameters.

Keywords: residual vibration, input shaping, flexible-joint manipulator, command shaping

\section{INTRODUCTION}

Motion control studies have become a key subject of robotics and other automation-related research areas. In the manufacturing industry, high-speed and sensitive motion control are necessary for highspeed and high quality production [1]. However, the requirement for high speed makes sensitive motion control difficult to accomplish due to residual vibrations. Therefore, finding a balance between the speed of motion and the elimination, or at least the reduction of, residual vibration has become an important part of the study of motion control and related practical applications [2] to [4].

When a force applied to a flexible mechanical system causes motion, it results in vibrations. Controlling the behaviour of such mechanical systems is generally difficult. In particular, the situation worsens if light and flexible components are used for a fast response. There are some studies relating to the control of the vibration of mechanical systems with flexible elements [5] to [7]. Command pre-shaping or input-shaping based methods have attracted the attention of some researchers [8] to [10]. A feedforward control is suggested as another input-shaping method, in which the shape of the command signal is altered to reduce system oscillations [11]. The initial works were conducted by Smith [12], whose technique is based on dividing a step input into two smaller steps, one of which is delayed in time. Next, the super-positioning of the step responses results in the cancellation of vibration on the system and provides a reduction in the settling time. However, there are some problems related to the robustness of the method due to uncertainties in the natural frequency and damping ratio.

Aspinwall [13] also suggested a different command-shaping approach. This method is based on shaping a rectangular or a 'bang-bang' forcing function. Another suggestion came from Meckl and Seering [14], who advised the construction of the input signal from either ramped sinusoids or versine functions. Due to the advantage of the rectangular function used in this method, the motion of the system is completed in a shortened period owing to the shape of the signal. Piazzi and Visioli [15] recently suggested a new technique based on shaping the input signal via inverse dynamic analysis. They proposed a polynomial function as a desired output to produce the input signal. Sahinkaya [16] and [17] suggested using a third order exponential function for the output motion to shape input signals using inverse dynamics.

Another approach to the problem is the use of an input-shaping technique that is based on convolving the reference command with a sequence of impulses [18] and [19]. This approach is reported in [20] to [22]. The increasing number of impulses used in the shaped signal leads to improvements in robustness to uncertainty in the system parameters. However, it also causes longer delays in system responses [23] and [24]. Magee and Book [25] suggested using an adaptive approach to implement a two-mode shaper.

In this study, a new residual vibration elimination method is presented. The benefit of the new technique is that it has a wider frequency range for which the results are insensitive to estimation errors of natural 
frequency. The input-shaping technique proposed in this study neither limits nor increases/decreases travelling time, i.e. without time limitation or time penalty. Due to their definition, most input-shaping methods, however, increase the travelling time by at least a half damped period or more [24], [26] and [27]. Further details on the comparison of methods of residual vibration elimination, such as zero vibration, zero vibration derivative, zero vibration double derivative and extra insensitive are provided by Singhose [7], Singhose et al. [27] and Vaughan et al. [28].

In the proposed approach, the distance to be travelled by the system is primarily divided into two equal parts. In the generation of each of the input signals, cycloid-plus-ramped and versine-plus-ramp functions are used [29]; then these two signals are joined to form the input signal applied to the system used in the elimination of residual vibration. Hence, a more robust method is obtained in reducing the residual vibrations. The proposed method has been applied in a flexible mechanical system. In the further sections of the presented work, the simulation and the experimental results of the new methods have been presented. In order to demonstrate the effectiveness of the proposed technique, the simulation and experimental results are compared with the results reported by Kapucu et al. [29]. The simulation and the experimental results show that the residual vibrations are considerably decreased with a high degree of robustness in the presence of uncertainty regarding the system parameters.

The outline of the paper is as follows: the first section presents the details of the experimental setup, which is followed by a section in which detail of the model of the system is provided. In the third section, the input-shaping methods are presented. In fourth section, the results related to computer simulation and experiments are presented. In the last section, concluding comments are made.

\section{EXPERIMENTAL SETUP}

The experimental setup used for performance analysis of the techniques is shown in Fig. 1. The experimental setup consists of a compound pendulum bonded to the sliding member (cart) fixed on the horizontal position of the hydraulically driven Stanford type manipulator, details of which are presented by Kapucu [29].

\section{MODELLING OF THE SYSTEM}

The mathematical model of the test setup is developed by using the Lagrange method. The model neglects the Coulomb friction of the joint and assumes that the manipulator is working in the horizontal plane and the pendulum in a vertical plane. Then, the equations of motion of the flexible system are [29]:

$$
\begin{gathered}
\left(M_{c}+m_{p}\right) \ddot{x}+m_{p} l \ddot{\theta} \cos \theta+c_{c} \dot{x}-m_{p} l \dot{\theta}^{2} \sin \theta= \\
=P_{1} A_{1}-P_{2} A_{2}, \\
m_{p} l \cos \theta \ddot{x}+m_{p} l \ddot{\theta}+c_{p} \dot{\theta}+m_{p} l g \sin \theta=0,
\end{gathered}
$$

where; $m_{p}$ is the mass of the pendulum (including the equivalent mass of the slender aluminium bar), $M_{c}$ the mass of sliding member (cart and translating link), $c_{c}$ the damping coefficient of the hydraulic cylinder, $c_{p}$ the damping coefficient of the pendulum, $A_{1}$ the piston area, $A_{2}$ the area of piston rod side, $P_{1}$ the pressure having impact on piston area, $P_{2}$ the pressure having impact on the area of the piston rod side.

The control system of the hydraulic cylinder and the servo valve is shown in Fig. 1. The generated force on the hydraulic piston is controlled by an electrohydraulic servo-valve. The control variable is the position of the spool of the servo valve that is defined by equations that are provided by Baysec and Jones [30].
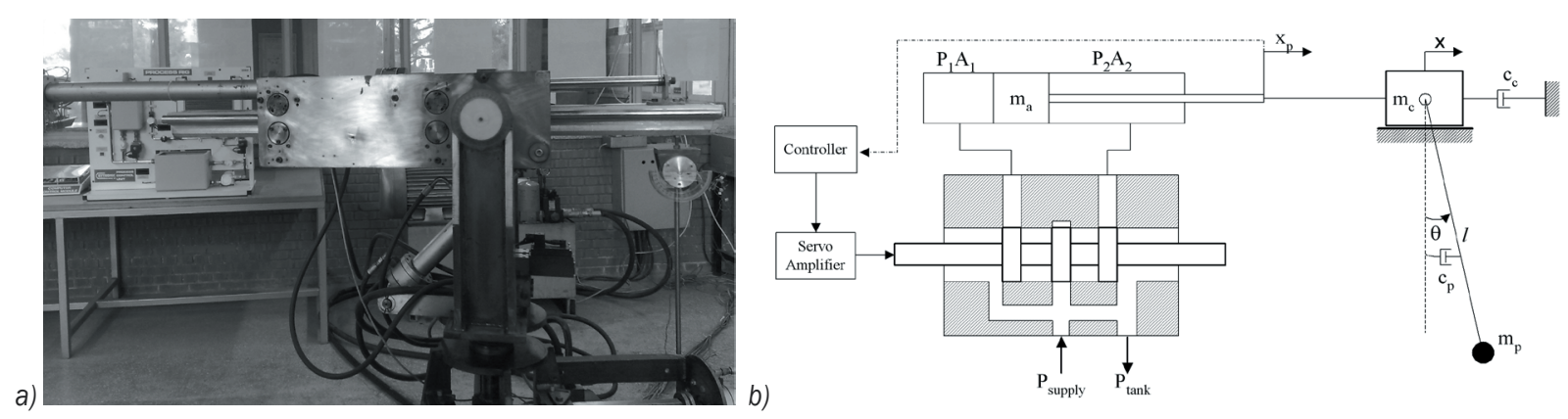

Fig. 1. a) The hydraulically activated cart and pendulum system; and b) its schematic illustration 


\section{INPUT-SHAPING METHODS}

The presented study deals with two different approaches used in the elimination of residual vibration. The first method (Method A) is proposed by Kapucu et al. [29], details of which are presented in Section 3.1. The second method (Method B) is the proposed new method, details of which are presented in Section 3.2.

\subsection{Method A: A Ramp-Plus-Ramped Cycloid-Plus-Ramped Versine-Based Reference Input Pre-Shaping Technique}

In this approach, the reference input is composed of three functions. The total distance to be covered within a specified time is divided into three parts. Each part is travelled by each of the three functions within the same travel time. Provided that the specified move time and the total distance are unchanged, vibration can be eliminated by adjusting the excursion distance of each function. Each component of the input creates such oscillations that they cancel each other out. This results in a reduction of residual vibration. Most elastic mechanical systems can be modelled with a second order differential equation.

The motion equation of such a system with damping ratio $\zeta$ and natural frequency $\omega_{n}$ can be defined as:

$$
\ddot{x}(t)+2 \zeta \omega_{n} \dot{x}(t)+\omega_{n}{ }^{2} x(t)=2 \zeta \omega_{n} \dot{y}(t)+\omega_{n}{ }^{2} y(t),
$$

where $\omega_{n}=\sqrt{k / m}$.

A motion profile of a cycloid-plus-ramped versine-plus-ramp function is expressed as:

$$
\begin{aligned}
Y & =\frac{L_{1} R t}{2 \pi}+\frac{L_{2}}{2 \pi}[R t-\sin (R t)]+\frac{L_{3} R t}{2 \pi}+ \\
& +\frac{L_{3}}{2 \pi}[1-\cos (R t)],
\end{aligned}
$$

where $L_{1}$ is the maximum excursion distance to be travelled by ramp motion profile, $L_{2}$ the maximum excursion distance to be travelled by cycloid motion profile, $L_{3}$ the maximum excursion distance to be travelled by ramped versine motion profile, $t$ time into motion, $\tau$ the travelling time, and $R=2 \pi / \tau$. Furthermore, the total distance can be written as $L=L_{1}+L_{2}+L_{3}$, then arranging the equation above becomes:

$$
Y(t)=\frac{L R t}{2 \pi}-\frac{L_{2}}{2 \pi} \sin (R t)+\frac{L_{3}}{2 \pi}(1-\cos (R t)) .
$$

The corresponding velocity profile is:

$$
\dot{Y}(t)=\frac{L R}{2 \pi}-\frac{L_{2} R}{2 \pi} \cos (R t)-\frac{L_{3} R}{2 \pi} \sin (R t) .
$$

The solution of the equation of motion, Eq. (3), under the effect of the positional input Eq. (5) and the corresponding velocity Eq. (6) yields the following excursion distance values $\left(L_{1}, L_{2}\right.$ and $\left.L_{3}\right)$ for zero residual vibration with zero initial conditions:

$$
\begin{gathered}
L_{1}=\frac{L R\left(R-2 \zeta \omega_{n}\right)}{\omega_{n}^{2}}=\frac{L T_{n}\left(T_{n}-2 \zeta \tau\right)}{\tau^{2}}, \\
L_{2}=L\left(1-\frac{R^{2}}{\omega_{n}^{2}}\right)=L\left(1-\frac{T_{n}^{2}}{\tau^{2}}\right), \\
L_{3}=\frac{2 L \zeta R}{\omega_{n}}=\frac{2 L \zeta T_{n}}{\tau}, \quad L=L_{1}+L_{2}+L_{3} .
\end{gathered}
$$

where $\omega_{n}$ is the natural frequency, and $T_{n}$ the natural frequency period. Variations of $L_{1}, L_{2}$ and $L_{3}$ are possible with traveling time $\tau$ to result in an oscillationfree displacement of the system. Theoretically, there is no traveling time restriction on the system, and this is the main advantage of this reference input-shaping technique [29].

\subsection{Method B: Proposed Input-Shaping Technique}

The proposed input-shaping method (Method B) utilises the first technique (Method A) presented in Section 3.1. With Method A, the Eqs. (4) to (7) are used to generate the command input required for the system. The proposed method, in contrast, divides the travelling time into two sections and calculates the command input as two separate inputs and then joins them to form the new input.

The calculations for the new method are as follows, where the input is divided into two sections, and each one is calculated independently to form the first and the second parts of the input signal.

$$
\begin{gathered}
L=L_{a}+L_{b}, \quad L_{a}=L_{b}=L / 2, \\
\tau=\tau_{a}+\tau_{b}, \quad \tau_{a}=\tau_{b}=\tau / 2, \\
R_{a, b}=2 \pi / \tau_{a, b}, \\
L_{1_{(a, b)}}=\frac{L R_{a, b}\left(R_{a, b}-2 \zeta \omega_{n}\right)}{\omega_{n}{ }^{2}}, \\
L_{2_{(a, b)}}=\frac{L\left(1-R_{a, b}\right)}{\omega_{n}{ }^{2}}, \quad L_{3_{(a, b)}}=\frac{2 L \zeta R_{a, b}}{\omega_{n}} .
\end{gathered}
$$




$$
Y=\left\{\begin{aligned}
0 \leq t \leq \frac{\tau}{2} \rightarrow Y & =\frac{L_{1 a} R_{a} t}{2 \pi}+\frac{L_{2 a}}{2 \pi}\left(R_{a} t-\sin \left(R_{a} t\right)\right)+ \\
& +\frac{L_{3 a} R_{a} t}{2 \pi}+\frac{L_{3 a}}{2 \pi}\left(1-\cos \left(R_{a} t\right)\right) \\
\frac{\tau}{2} \leq t \leq \tau \rightarrow Y & =\frac{L_{1 b} R_{b} t}{2 \pi}+\frac{L_{2 b}}{2 \pi}\left(R_{b} t-\sin \left(R_{b} t\right)\right)+ \\
& +\frac{L_{3 b} R_{b} t}{2 \pi}+\frac{L_{3 b}}{2 \pi}\left(1-\cos \left(R_{b} t\right)\right)
\end{aligned}\right\} .
$$

As defined in Eqs. (8) to (12), the new method allows a virtual division of the motion of the system into two steps. Because the first step is completed with almost steady motion and with relatively reduced vibration levels, the second part of the motions starts with the advantage of very little or almost no residual vibrations. Consequently, the result of the second part of the motion yields better performance relative to the previously mentioned method.

The details provided in Fig. 2 illustrate the command inputs generated using two different methods considered for analysis. The details on the generation of input-shaping functions for Methods A and B can be found in Sections 3.1 and 3.2, respectively.

As illustrated in Fig. 2, Method A consists of two components with which the resulting residual vibration cancels out due to reverse act. However, Method B consists of four parts resulting in eliminated residual vibration at half way to the destination position. These features of the input signals can be seen clearly in Fig. 2.

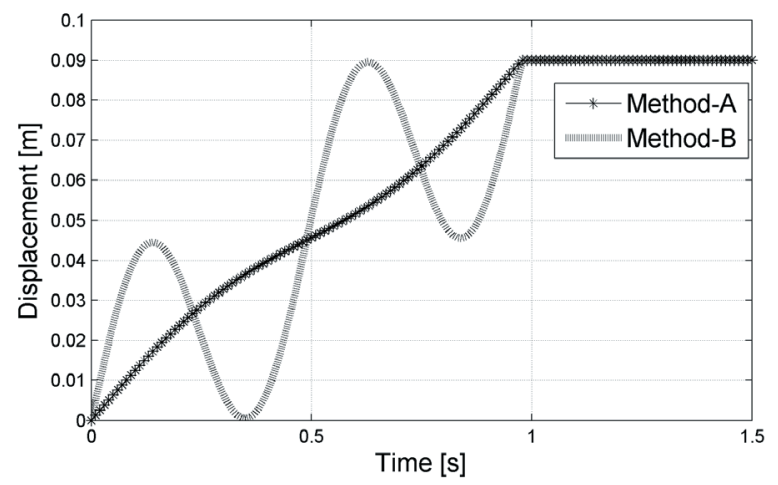

Fig. 2. The input-shaping methods and generated input commands for total travelling distance of $L=0.09 \mathrm{~m}$ and travelling time $\tau=0.9703 \mathrm{~s}\left(R_{a, b}=12.8503, L_{1 a, b}=2.7508, L_{2 a, b}=-2.2680\right.$,

$$
\left.L_{3 a, b}=0.0172\right)
$$

\section{SIMULATION AND EXPERIMENTS}

In order to perform a comparison amongst the inputshaping methods and the experimental results of the techniques, the previously described experimental setup and the mathematical model of the system is used. The experimental setup is driven using each input and the resulting residual vibrations as well as the cart position are measured. The Matlab model of the system is also provided with the same input commands to demonstrate the correlation between the theoretical and experimental results obtained.

For the randomly selected motion time $\tau=0.9703 \mathrm{~s}$ and the travel distance of the hydraulic cylinder $L=0.09 \mathrm{~m}$, the generated input commands are applied to the Matlab model of the system and the experimental setup. The results of the study are presented in Figs. 3 to 5 for Methods A, B. In Figs. 3 to $5, \omega_{n}{ }^{*}$ represents the estimated values the natural frequencies of the system.

In Figs. 3 to 5, the reference input and displacement of the hydraulic cylinder are given in meters. In contrast, the pendulum oscillation is given in the unit of radians. It can be seen that the simulation and test results match up very closely. This simply indicates that the behaviour of the developed Matlab model and the experimental setup are very much the same. This is mainly due to the accurate mathematical model of the mechanical [29] and the hydraulic systems [30]. In Figs. 3 to 5, simulation and experimental results of Methods A and B are illustrated. In these figures, an estimation error of $\pm 25 \%$ is introduced to the natural frequency of the system used in the calculation of the input signals. Figs. 3 to 5 are plotted with estimation errors of -25 , $0,+25 \%$, respectively. In Figs. 3 to 5, it can be seen that Method A, the estimation error causes increasing residual vibrations of the pendulum ranging from $4.68 \times 10^{-4}$ to $1.92 \times 10^{-2} \mathrm{rad}$. It can be seen that increase error in estimation of the natural frequency of the system causes increasing residual vibrations. These results are also validated in Fig. 6 (Method A) and Fig. 7 (Method A) where different predicted natural frequencies and sensitivity curves are presented, respectively.

In Figs. 3 to 5, however, the results differ, ranging from $3.195 \times 10^{-3}$ to $1.23 \times 10^{-2} \mathrm{rad}$. As can be seen from Figs. 3 to 5, the increase in estimation of the natural frequency of the system causes much less increase in residual vibrations. Therefore, it can be concluded that the proposed new method is better in comparison to the old method. This conclusion is much clearer from the Fig. 7 (Method B) where the sensitivity curve for the new method is provided. The proposed new method has a wider insensitivity to variation of natural frequency resulting in a wider spectra compared to the old method. 
a)

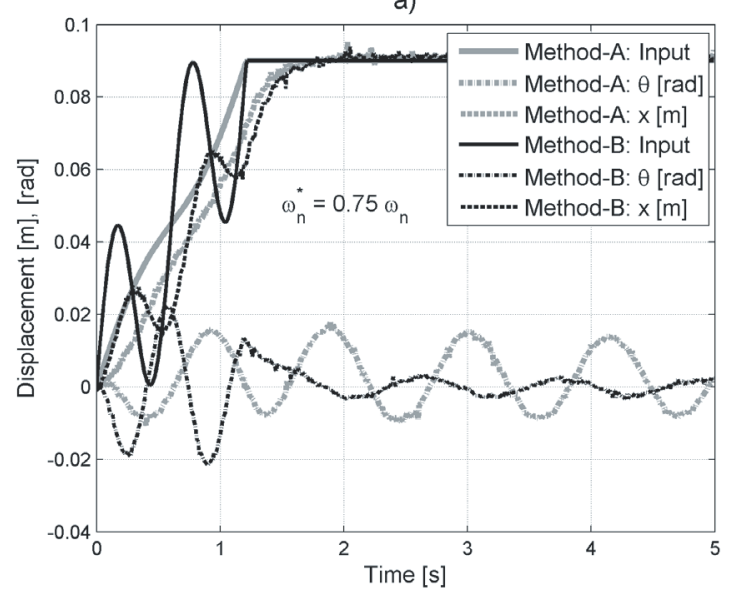

b)

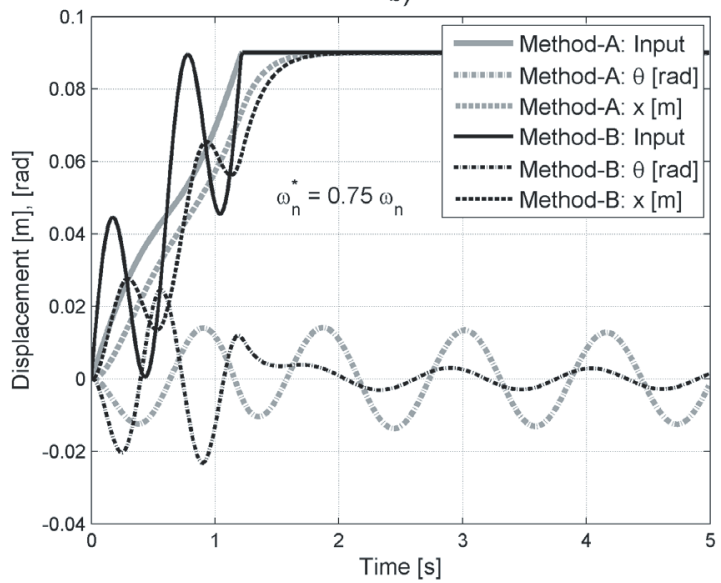

Fig. 3. Method $A$ and Method $B$ with a) experimental and b) simulation results for total travelling distance of $L=0.09 \mathrm{~m}$ and travelling time $\tau=1.293 \mathrm{~s}$ for $-25 \%$ estimation error of natural frequency

a)

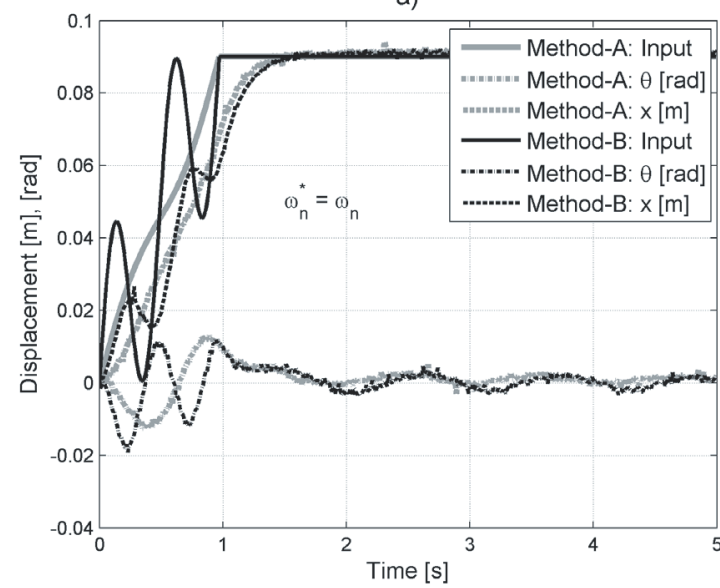

b)

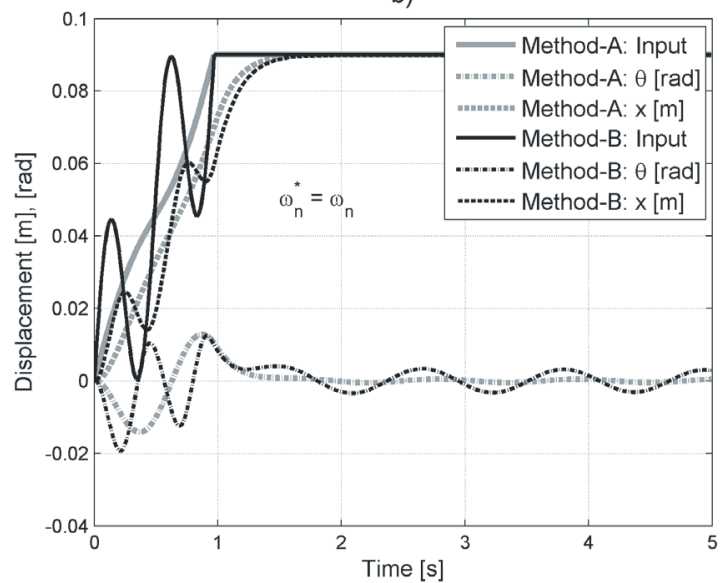

Fig. 4. Method $A$ and Method $B$ with a) experimental and b) simulation results for total travelling distance of $L=0.09 \mathrm{~m}$ and travelling time $\tau=0.9703 \mathrm{~s}$ for accurate estimation of natural frequency

a)

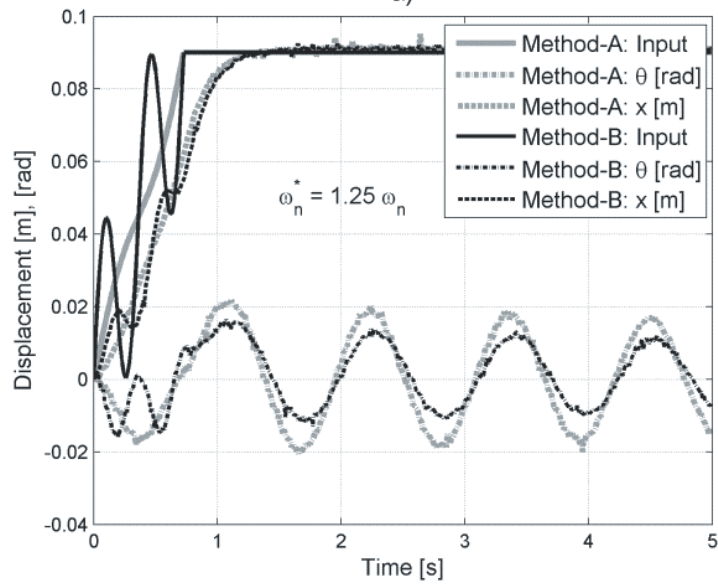

b)

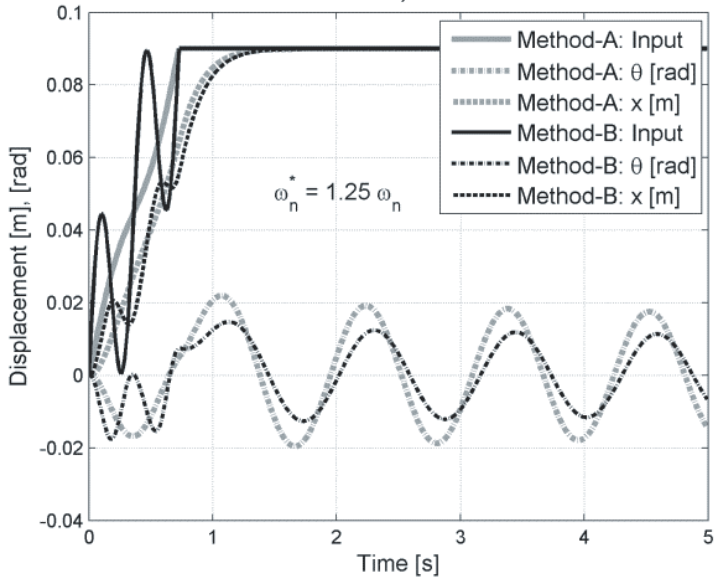

Fig. 5. Method $A$ and Method $B$ with a) experimental and b) simulation results for total travelling distance of $L=0.09 \mathrm{~m}$ and travelling time $\tau=0.776 \mathrm{~s}$ for $+25 \%$ estimation error of natural frequency 
Method-A

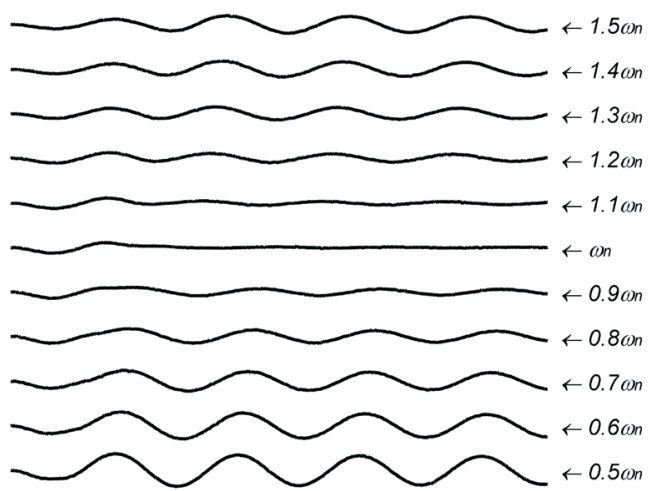

Method-B

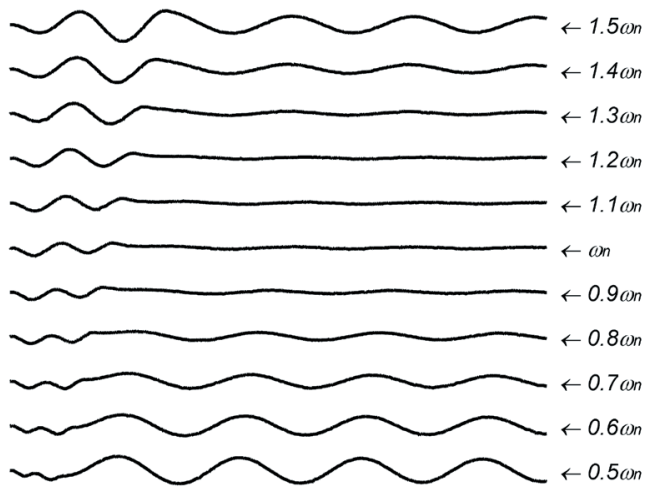

Fig. 6. Pendulum positions of different predicted natural frequencies for input-shaping Methods $A$ and B-related experimental results for total travelling distance of $L=0.09 \mathrm{~m}$, travelling time $\tau=0.9703 \mathrm{~s}$

a)

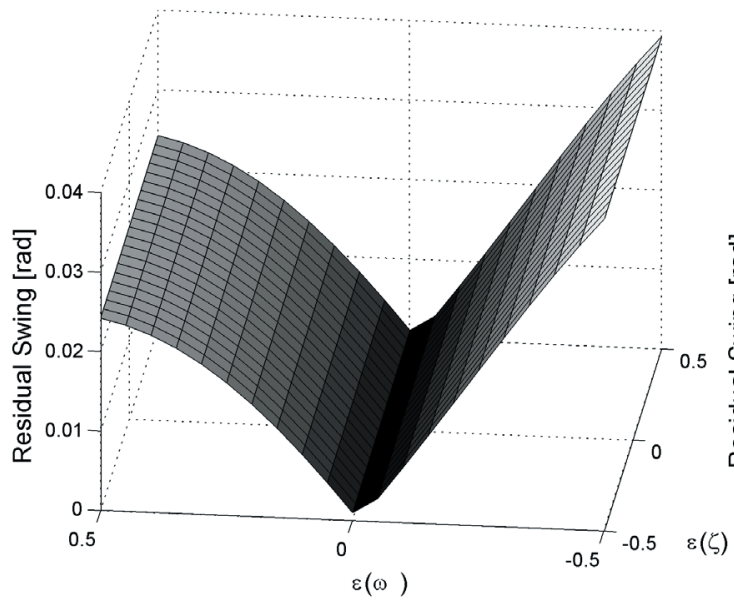

b)

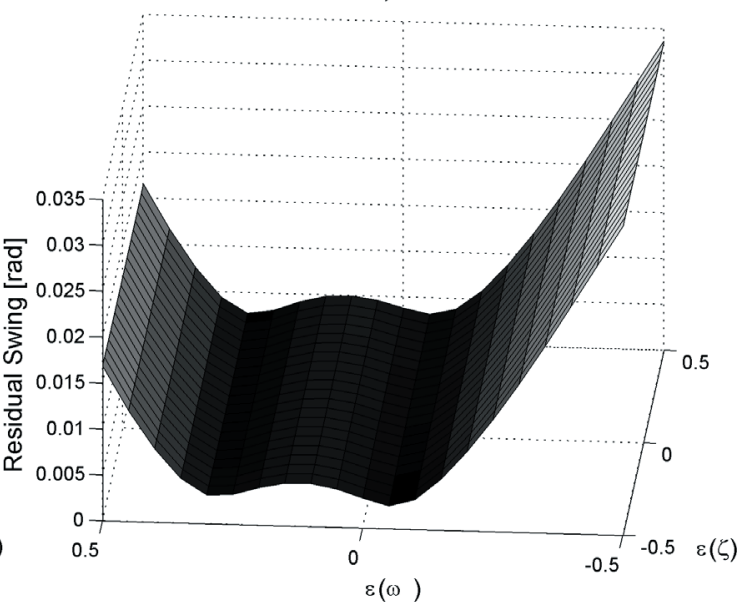

Fig. 7. Robustness of the system to uncertainties in the mode frequencies and damping ratios for the studied; a) Method A and b) Method B

In order to estimate the robustness of the proposed methods against estimation error of the natural frequency of the model parameters, an error function has been defined as:

$$
\varepsilon\left(\omega_{n}\right)=\frac{\omega_{n}}{\omega_{n}{ }^{*}}-1,
$$

where, $\omega_{n}, \omega_{n}{ }^{*}$ shows actual and predicted values the natural frequencies of system, respectively.

Due to the very low level of the damping ratio of the system $(\zeta=0.08)$, its variation due to estimation error does not seem to affect the results as shown in Fig. 7. Therefore, the robustness analysis is performed only for the natural frequency estimation error-related variations.
In Fig. 7, the variation of the residual vibration is presented against estimation error in the natural frequency and damping ratio. It can clearly be seen that the variation of error or uncertainty of damping ratio has a relatively reduced effect on the residual vibration of the system. Therefore, the uncertainties of the damping ratio do not play an important role in affecting the behaviour of the system due to its very low value, $\zeta=0.08$. In contrast, the estimation error in the natural frequency appears to affect the motion of the system and the resulting residual vibration levels.

The figure also shows that the proposed method provides a more robust response relative to Method A. It is noteworthy that the lowest level of residual vibration is achieved using the proposed new technique. 


\section{CONCLUSION}

In this study, a new residual vibration elimination method is introduced, and a comparative study of two input-shaping techniques is performed. Both the theoretical and practical results have shown that the new technique seems to perform better relative to the other method mentioned.

- From the simulation and experimental results, it is possible to conclude the following:

- It is shown that the proposed method (Method B) is far more robust than the old method (Method A). In other words, the proposed new method has a relatively wider insensitive natural frequency spectra in comparison to the old one.

- The advantage of the proposed technique is that it neither limits nor increases the move time, i.e. no time limitation or time penalty. Most conventional input-shaping methods, however, tend to increase the travelling time by at least a half damped period or more.

It is shown that proposed new technique is simple and easy to implement, and can be considered to be a versatile and effective way to determine a trajectory resulting in reduced or eliminated residual vibrations of flexible systems with high robustness.

Therefore, it can be concluded that the proposed new method (Method B) is better relative to the old one (Method A) and to other conventional methods.

\section{REFERENCES}

[1] Radoičić, G., Jovanović, M. (2013). Experimental identification of overall structural damping of system. Strojniški vestnik - Journal of Mechanical Engineering, vol. 58, no. 4, p. 260-268, DOI:10.5545/ sv-jme.2012.569.

[2] Changa, P.H., Park, H.S. (2005). Time-varying input shaping technique applied to vibration reduction of an industrial robot. Control Engineering Practice, vol. 13, no. 1 p. 121-130, DOI:10.1016/j. conengprac.2004.02.009.

[3] Jokić, M., Stegić, M., Kranjčević, N. (2012). Structural stiffness optimization with respect to vibration response. Transactions of FAMENA, vol. 36, no. 2, p. $1-8$.

[4] Golubović, Z., Lekić, Z., Jović, S. (2012). Influence of bucket wheel vertical vibration on bucket wheel excavator (BWE) digging force. Tehnički vjesnik Technical Gazette, vol. 19, no. 4, p. 807-812.

[5] Singhose, W., Pao, L. (1997). A comparison of input shaping and time-optimal flexible-body control. Control Engineering Practice, vol. 5, no. 4, p. 459-467, DOI:10.1016/S0967-0661(97)00025-7.
[6] Shan, J., Liu, H., Sun, D. (2005). Modified input shaping for a rotating single-link flexible manipulator. Journal of Sound and Vibration, vol. 285, p. 187-207, DOI:10.1016/j.jsv.2004.08.035.

[7] Singhose, W. (2009). Command shaping for flexible systems: A review of the first 50 years. International Journal of Precision Engineering and Manufacturing, vol. 10, no. 4, p. 153-168, DOI:10.1007/s12541-0090084-2.

[8] Raubar, E., Vrančić, D. (2012). Anti-sway system for ship-to-shore cranes. Strojniški vestnik - Journal of Mechanical Engineering, vol. 58, no. 5, p. 338-344, DOI:10.5545/sv-jme.2010.127.

[9] Singhose, W., Porter, L., Kenison, M., Kriikku, E. (2000). Effects of hoisting on the input shaping control of gantry cranes. Control Engineering Practice, vol. 8, no. 10, p. 1159-1165, DOI:10.1016/S09670661(00)00054-X.

[10] Changa, P.H., Park, J. (2001). A concurrent design of input shaping technique and a robust control for highspeed/high-precision control of a chip mounter. Control Engineering Practice, vol. 9, no. 12, p. 1279-1285, DOI:10.1016/S0967-0661(01)00092-2.

[11] Kim, D., Singhose, W. (2010). Performance studies of human operators driving double-pendulum bridge cranes. Control Engineering Practice, vol. 18, no. 6, p. 567-576, DOI:10.1016/j.conengprac.2010.01.011.

[12] Smith, O.J.M. (1958). Feedback Control Systems, McGraw-Hill, New York.

[13] Aspinwall, D.M. (1980). Acceleration profiles for minimizing residual response. ASME Journal of Dynamic Systems, Measurement and Control, vol. 102, no. 1, p. 3-6, DOI:10.1115/1.3140620.

[14] Meckl, P.H., Seering, W. (1990). Experimental evaluation of shaped inputs to reduce vibration for a Cartesian robot. ASME Journal of Dynamic Systems, Measurement and Control, vol. 112, no. 2, p. 159-165, DOI:10.1115/1.2896122.

[15] Piazzi, A., Visioli, A. (2000). Minimum-time systeminversion- based motion planning for residual vibration reduction. IEEE/ASME Transactions on Mechatronics, vol. 5, no. 1, p. 12-22, DOI:10.1109/3516.828585.

[16] Sahinkaya, M.N. (2001). Input shaping for vibrationfree positioning of flexible systems. Proceedings of the Institution of Mechanical Engineers, Part I, p. 467-481.

[17] Sahinkaya, M.N. (2004). Exponential input shaping for vibration free positioning of lightly damped multimode systems. $7^{\text {th }}$ International Conference on Motion and Vibration Control, p. 8-11.

[18] Mimmi, G., Pennacchi, P. (2001). Pre-shaping motion input for a rotating flexible link. International Journal of Solids and Structures, vol. 38, no. 10-13, p. 20092023, DOI:10.1016/S0020-7683(00)00149-9.

[19] Cutforth, C.F., Pao, L.Y. (2004). Adaptive input shaping for maneuvering flexible structures. Automatica, vol. 40, no. 4, p. 685-693, DOI:10.1016/j. automatica.2003.11.013. 
[20] Park, J.Y., Chang, P.H. (2004). Vibration control of a telescopic handler using time delay control and commandless input shaping technique. Control Engineering Practice, vol. 12, no. 6, p. 769-780, DOI:10.1016/j.conengprac.2003.09.005.

[21] Gurleyuk, S.S., Cinal, S. (2007). Robust threeimpulse sequence input shaper design. Journal of Vibration and Control, vol. 13, no. 12, p. 1807-1818, DOI:10.1177/1077546307080012.

[22] Gurleyuk, S.S., Hacioglu, R., Cinal, S. (2007). Threestep input shaper for damping tubular step motor vibrations. Journal of Mechanical Engineering Science, vol. 221, no. 1, p. 1-9, DOI:10.1243/0954406JMES381.

[23] Singer, N.C. (1989). Residual Vibration Reduction in Computer Controlled Machines. PhD thesis, MIT Artificial Intelligence Laboratory, Massachusetts.

[24] Singer, N.C., Seering, W.P. (1990). Preshaping command inputs to reduce system vibration. ASME Journal of Dynamic Systems, Measurement and Control, vol. 112, p. 76-82, DOI:10.1115/1.2894142.

[25] Magee, D.P., Book W.J. (1993). Implementing modified command filtering to eliminate multiple modes of vibration. Proceedings of the American Control Conference, p. 2700-2704.

[26] Singhose, W., Seering, W., Singer, N. (1994). Residual vibration reduction using vector diagrams to generate shaped inputs. Journal of Mechanical Design, vol. 116, no. 2, p. 654-659, DOI:10.1115/1.2919428.

[27] Singhose, W., Porter, L., Singer, N. (1995). Vibration reduction using multi-hump extra-insensitive input shapers. American Control Conference, p. 3830-3834.

[28] Vaughan, J., Yano, A., Singhose, W. (2008). Comparison of robust input shapers. Journal of Sound and Vibration, vol. 315, no. 4-5, p. 797-815, DOI:10.1016/j.jsv.2008.02.032.

[29] Kapucu, S., Yildirim, N., Yavuz, H., Baysec, S. (2008). Suppression of residual vibration of a translatingswinging load by a flexible manipulator. Mechatronics, vol. 18, no. 3, p. 121-128, DOI:10.1016/j. mechatronics.2007.10.007.

[30] Baysec, S., Jones, J.R. (1987). An improved model of an electro hydraulic servo valve. Proceedings of 7 th IFToMM Congress, p. 1489-1494. 